\title{
Numerical Method for Electromagnetic Wave Propagation Problem in a Cylindrical Anisotropic Waveguide with Longitudinal Magnetization
}

\author{
Eugene Smolkin* and Maxim Snegur \\ Penza State University, Penza, Russia \\ *corresponding author, E-mail: e.g.smolkin@hotmail.com
}

\begin{abstract}
The propagation of monochromatic electromagnetic waves in metal circular cylindrical dielectric waveguide with longitudinal magnetization filled with anisotropic inhomogeneous waveguide is considered. The physical problem is reduced to solving a transmission eigenvalue problem for a system of ordinary differential equations. Spectral parameters of the problem are propagation constants of the waveguide. Numerical results are obtained using a modification of the projecting methods. The comparison with known exact solutions (for particular values of parameters) are made.
\end{abstract}

\section{Introduction}

A large class of vector electromagnetic problem concerns electromagnetic wave propagation. The constitutive parameters $\varepsilon$ and $\mu$ of standard dielectric and magnetic media are determined by their physical structure. However, the media with unusual properties are often required which can be obtained using either dielectrics that are uniform or partially filled. The parameters of such media depend on the mutual position of the particles and may be anisotropic [1]. It is known also that the permittivity of a dielectric (or the permeability of a magnetic) may depend on the radial coordinate [2]. The primary goal here is to construct a numerical method to determine the spectrum of normal electromagnetic waves that propagate in such structures.

Numerical methods for calculating the parameters of various types of waveguide structures are described in the monographs and review papers $[3,4,5,6]$. However, it should be said that most of the methods applied to homogeneous waveguides, are not common and are difficult to implement and apply for specific inhomogeneous and/or anisotropic structures.

In this work the wave propagation in inhomogeneous metal-dielectric anisotropic cylindrical waveguides is studied numerically using the modification of the projection methods [7].

\section{Statement of the problem}

Consider three-dimensional space $\mathbb{R}^{3}$ with a cylindrical coordinate system $O \rho \varphi z$ filled with isotropic medium having constant permittivity $\varepsilon=\varepsilon_{0}\left(\varepsilon_{0}>0\right.$ is the permittivity of free space), and constant permeability $\mu=\mu_{0}$ ( where $\mu_{0}>0$ is the permeability of free space).
A metal dielectric circular cylindrical waveguide $\Sigma$ filled with anisotropic inhomogeneous medium is placed parallel to the axis $O z$. The waveguide $\Sigma$ has a cross section

$$
\Sigma:=\left\{(\rho, \varphi, z): r_{0} \leq \rho \leq r, 0 \leqslant \varphi<2 \pi\right\}
$$

and its generating line (the waveguide axis) is parallel to the axis $O z$ (see. Fig. 1).

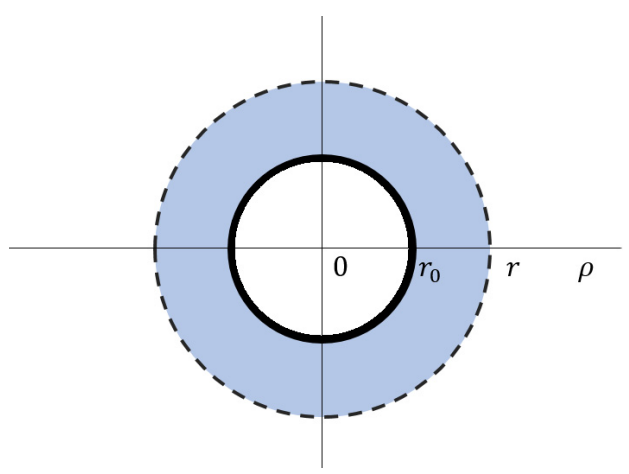

Figure 1: The cylindrical anisotropic waveguide $\Sigma$.

We will consider monochromatic waves

$$
\begin{aligned}
& \mathbf{E} e^{-i \omega t}=e^{-i \omega t}\left(E_{\rho}, E_{\varphi}, E_{z}\right)^{T}, \\
& \mathbf{H} e^{-i \omega t}=e^{-i \omega t}\left(H_{\rho}, H_{\varphi}, H_{z}\right)^{T},
\end{aligned}
$$

where $(\cdot)^{T}$ denotes the transpose operation. Each component of the field $\mathbf{E}, \mathbf{H}$ is a function of three spatial variables.

Complex amplitudes of the electromagnetic field $\mathbf{E}, \mathbf{H}$ satisfy the Maxwell equations

$$
\left\{\begin{array}{l}
\operatorname{rot} \mathbf{H}=-\mathrm{i} \omega \varepsilon \mathbf{E}, \\
\operatorname{rot} \mathbf{E}=\mathrm{i} \omega \mu \mathbf{H},
\end{array}\right.
$$

subject to the following boundary conditions. The tangential components of the electric field vanish on the metal surface $\rho=r_{0}$; tangential field components are continuous on the media interface $\rho=r$; the complex amplitudes obey the radiation condition at infinity: the electromagnetic field decays as $O\left(|\rho|^{-1}\right)$ when $\rho \rightarrow \infty$. The permittivity $\varepsilon$ inside the waveguide is constant; the permeability $\hat{\mu}$ is specified by the expression

$$
\hat{\mu}=\left[\begin{array}{ccc}
\mu_{\rho} & -i \mu_{\varphi} & 0 \\
i \mu_{\varphi} & \mu_{\rho} & 0 \\
0 & 0 & \mu_{z}
\end{array}\right]
$$


where $\mu_{\rho}(\rho), \mu_{\varphi}(\rho)$ and $\mu_{z}(\rho)$ are sufficiently smooth functions which depend on the radial coordinate $\rho$.

The surface waves propagating along the axis $O z$ of the waveguide $\Sigma$ have the form [8]

$$
\begin{aligned}
& E_{\rho}=\mathrm{E}_{\rho}(\rho) e^{i \gamma z}, \quad H_{\rho}=\mathrm{H}_{\rho}(\rho) e^{i \gamma z} \\
& E_{\varphi}=\mathrm{E}_{\varphi}(\rho) e^{i \gamma z}, \\
& E_{\varphi}=\mathrm{E}_{z}(\rho) e^{i \gamma z},
\end{aligned}
$$

where $\gamma$ is the real propagation constant (spectral parameter of the problem). In what follows we often omit the arguments of functions when it does not lead to misunderstanding.

\section{Differential equations}

Inside the waveguide $\mu=\hat{\mu}$ and $\varepsilon=\varepsilon$. Substituting $\mathbf{E}$ and $\mathbf{H}$ with components (3) into equations (1), we obtain

$$
\left\{\begin{aligned}
i \gamma H_{\varphi} & =i \omega \varepsilon E_{\rho} \\
i \gamma H_{\rho}-H_{z}^{\prime} & =-i \omega \varepsilon E_{\varphi} \\
\frac{1}{\rho}\left(\rho H_{\varphi}\right)^{\prime} & =-i \omega \varepsilon E_{z} \\
i \gamma E_{\varphi} & =-i \omega \mu_{\rho} H_{\rho}-\omega \mu_{\varphi} H_{\varphi} \\
i \gamma E_{\rho}-E_{z}^{\prime} & =-\omega \mu_{\varphi} H_{\rho}+i \omega \mu_{\rho} H_{\varphi} \\
\frac{1}{\rho}\left(\rho E_{\varphi}\right)^{\prime} & =i \omega \mu_{z} H_{z}
\end{aligned}\right.
$$

where the prime denotes differentiation w.r.a $\rho$. Expressing the functions $E_{\rho}, E_{z}, H_{\rho}$ and $H_{z}$ through $E_{\varphi}$ and $H_{\varphi}$ from the $1 \mathrm{st}, 3 \mathrm{rd}$, 4th and 6th equation of system (4), we find

$$
\begin{array}{ll}
H_{\rho}=\frac{-\gamma E_{\varphi}+\omega \mu_{\varphi} i H_{\varphi}}{\omega \mu_{\rho}}, & E_{\rho}=\frac{\gamma H_{\varphi}}{\omega \varepsilon}, \\
H_{z}=-\frac{\left(\rho i E_{\varphi}\right)^{\prime}}{\omega \mu_{z} \rho}, & E_{z}=\frac{\left(\rho i H_{\varphi}\right)^{\prime}}{\omega \varepsilon \rho} .
\end{array}
$$

Substituting the expressions for $E_{\rho}, E_{z}, H_{\rho}$ and $H_{z}$ into the 2 nd and 5 th equations of system (5) and introducing the notation $u_{e}:=i \rho E_{\varphi}(\rho), u_{m}:=\rho H_{\varphi}(\rho)$, we obtain

$$
\begin{aligned}
L_{e} u_{e} & =u_{e}^{\prime \prime}-p_{e} u_{e}^{\prime}+\left(q_{e}-h_{e} \gamma^{2}\right) u_{e}=\gamma f_{e} u_{m}, \\
L_{m} u_{m} & =u_{m}^{\prime \prime}-p_{m} u_{m}^{\prime}+\left(q_{m}-h_{m} \gamma^{2}\right) u_{m}=\gamma f_{m} u_{e}
\end{aligned}
$$

where

$$
\begin{gathered}
p_{e}=\frac{\left(\rho \mu_{z}\right)^{\prime}}{\rho \mu_{z}}, \quad p_{m}=\frac{1}{\rho}, \\
q_{e}=\omega^{2} \varepsilon \mu_{z}, \quad q_{m}=\omega^{2} \varepsilon \frac{\mu_{\rho}^{2}-\mu_{\varphi}^{2}}{\mu_{\rho}}, \\
h_{e}=\frac{\mu_{z}}{\mu_{\rho}}, \quad h_{m}=1, \\
f_{e}=\omega \frac{\mu_{\varphi} \mu_{z}}{\mu_{\rho}}, \quad f_{m}=\omega \frac{\varepsilon \mu_{\varphi}}{\mu_{\rho}} .
\end{gathered}
$$

Outside the waveguide, where $\mu=\mu_{0}=1$ and $\varepsilon=$ $\varepsilon_{0}=1$, system (1) takes the form of Bessel's equations

$$
\begin{aligned}
\left(\frac{u_{e}^{\prime}}{\rho}\right)^{\prime}-\frac{k_{1}^{2} u_{e}}{\rho} & =0, \\
\left(\frac{u_{m}^{\prime}}{\rho}\right)^{\prime}-\frac{k_{1}^{2} u_{m}}{\rho} & =0
\end{aligned}
$$

with general solutions

$$
\begin{aligned}
u_{e} & =C_{1} \rho I_{1}\left(k_{1} \rho\right)+C_{2} \rho K_{1}\left(k_{1} \rho\right), \rho>r \\
u_{m} & =C_{3} \rho I_{1}\left(k_{1} \rho\right)+C_{4} \rho K_{1}\left(k_{1} \rho\right), \rho>r
\end{aligned}
$$

where $k_{1}^{2}=\gamma^{2}-\omega^{2}, I_{1}$ is the modified Bessel function and $K_{1}$ is the Macdonald function [9] and $C_{1}, \ldots, C_{4}$ denote arbitrary constants.

The solutions (7) takes a form

$$
\begin{array}{r}
u_{e}=C_{1} \rho K_{1}\left(k_{1} \rho\right), \rho>r \\
u_{m}=C_{2} \rho K_{1}\left(k_{1} \rho\right), \rho>r
\end{array}
$$

where the radiation condition at infinity is taken into account.

\section{Transmission conditions and transmission problem}

Tangential components of the electromagnetic field are known to be continuous at the interface. In this case the tangential components are $E_{\varphi}, E_{z}, H_{\varphi}$ and $H_{z}$. Thus we obtain the following transmission conditions for $u_{e}$ and $u_{m}$

$$
\begin{gathered}
u_{e}\left(r_{0}\right)=0, u_{m}^{\prime}\left(r_{0}\right)=0, \\
u_{e}(r-0)=u_{e}(r+0), u_{m}(r-0)=u_{m}(r+0), \\
\frac{u_{e}^{\prime}(r-0)}{\mu_{z}(r-0)}=\frac{u_{e}^{\prime}(r+0)}{\mu_{0}}, \frac{u_{m}^{\prime}(r-0)}{\varepsilon}=\frac{u_{m}^{\prime}(r+0)}{\varepsilon_{0}}
\end{gathered}
$$

where $\left.[v]\right|_{\rho=s}=\lim _{\rho \rightarrow s-0} v(\rho)-\lim _{\rho \rightarrow s+0} v(\rho)$ is the jump of the limit values of the function at the point $s$.

The main problem considered in this study is formulated as follows: Problem P: to find $\hat{\gamma}$ such that there exist non-trivial functions $u_{e}(\rho ; \hat{\gamma})$ and $u_{m}(\rho ; \hat{\gamma})$ satisfying system (6), transmission conditions (9), and having the form (8) outside the waveguide. 


\section{Variation formulation}

Let us give the variational formulation of the problem $P$. Using the first Green's formula, we obtain

$$
\begin{gathered}
\int_{r_{0}}^{r} v L u d \rho= \\
=\int_{r_{0}}^{r} v u^{\prime \prime} d \rho-\int_{r_{0}}^{r} v p u^{\prime} d \rho+\int_{r_{0}}^{r} v\left(q-h \gamma^{2}\right) u d \rho= \\
=\left.u^{\prime} v\right|_{r_{0}} ^{r}-\int_{r_{0}}^{r} u^{\prime} v^{\prime} d \rho-\int_{r_{0}}^{r} p u^{\prime} v d \rho+\int_{r_{0}}^{r}\left(q-h \gamma^{2}\right) u v d \rho= \\
=-\gamma^{2} \int_{r_{0}}^{r} h u v d \rho-\int_{r_{0}}^{r} u^{\prime} v^{\prime} d \rho- \\
-\int_{r_{0}}^{r} p u^{\prime} v d \rho+\int_{r_{0}}^{r} q u v d \rho+u^{\prime}(r) v(r), \quad \text { (10) }
\end{gathered}
$$

where $u=u_{j}, h=h_{j}, p=p_{j}, q=q_{j}, j=e$ or $m$.

Let us consider the smooth test functions $v_{e}$ and $v_{m}$.

Note 1. We assume that the test functions $v_{e}$ and $v_{m}$ satisfy the following conditions

$$
\begin{gathered}
v_{e}\left(r_{0}\right)=0, \quad v_{e}(r)=1, \\
v_{m}^{\prime}\left(r_{0}\right)=0, \quad v_{m}(r)=1,
\end{gathered}
$$

which coincide with conditions for functions $u_{e}$ and $u_{m}$ at the boundary $r_{0}$.

Multiplying the left and right sides of equations (10) by the test functions $v_{e}$ and $v_{m}$, respectively, and summing up we obtain

$$
\begin{gathered}
\int_{r_{0}}^{r}\left(v_{e} L_{e} u_{e}+v_{m} L_{m} u_{m}\right) d \rho= \\
=-\gamma^{2} \int_{r_{0}}^{r}\left(h_{e} u_{e} v_{e}+h_{m} u_{m} v_{m}\right) d \rho- \\
-\int_{r_{0}}^{r}\left(u_{e}^{\prime} v_{e}^{\prime}+u_{m}^{\prime} v_{m}^{\prime}\right) d \rho-\int_{r_{0}}^{r}\left(p_{e} u_{e}^{\prime} v_{e}+u_{m}^{\prime} v_{m}\right) d \rho+ \\
+\int_{r_{0}}^{r}\left(q_{e} u_{e} v_{e}+q_{m} u_{m} v_{m}\right) d \rho+ \\
+u_{e}^{\prime}(r) v_{e}(r)+u_{m}^{\prime}(r) v_{m}(r) .
\end{gathered}
$$

Taking into account the right-hand sides of the equations of system (6), we have

$$
\begin{aligned}
\int_{r_{0}}^{r}\left(v_{e} L_{e} u_{e}+v_{m} L_{m} u_{m}\right) d \rho= \\
=\gamma \int_{r_{0}}^{r}\left(f_{e} u_{m} v_{e}+f_{m} u_{e} v_{m}\right) d \rho .
\end{aligned}
$$

From (9) we determine $u_{e}^{\prime}$ and $u_{m}^{\prime}$ at the point $r$

$$
\begin{gathered}
u_{e}^{\prime}(r)=-k_{1} \frac{\mu_{z}(r)}{\mu_{0}} \frac{K_{0}\left(k_{1} r\right)}{K_{1}\left(k_{1} r\right)} u_{e}(r), \\
u_{m}^{\prime}(r)=-k_{1} \frac{\varepsilon}{\varepsilon_{0}} \frac{K_{0}\left(k_{1} r\right)}{K_{1}\left(k_{1} r\right)} u_{m}(r) .
\end{gathered}
$$

From (11) taking into account (12) and (13), we obtain the variational equation

$$
\begin{aligned}
& \gamma^{2} \int_{r_{0}}^{r}\left(h_{e} u_{e} v_{e}+h_{m} u_{m} v_{m}\right) d \rho+ \\
& +\int_{r_{0}}^{r}\left(u_{e}^{\prime} v_{e}^{\prime}+u_{m}^{\prime} v_{m}^{\prime}\right) d \rho+\int_{r_{0}}^{r}\left(p_{e} u_{e}^{\prime} v_{e}+p_{m} u_{m}^{\prime} v_{m}\right) d \rho- \\
& \quad-\int_{r_{0}}^{r}\left(q_{e} u_{e} v_{e}+q_{m} u_{m} v_{m}\right) d \rho+ \\
& \left.+k_{1} \frac{K_{0}\left(k_{1} r\right)}{K_{1}\left(k_{1} r\right)}{ }_{\mu_{z}(r)}^{\mu_{0}} u_{e}(r) v_{e}(r)+\frac{\varepsilon}{\varepsilon_{0}} u_{m}(r) v_{m}(r)\right)+ \\
& +\gamma \int_{r_{0}}^{r}\left(f_{e} u_{m} v_{e}+f_{m} u_{e} v_{m}\right) d \rho, \forall v_{e}, v_{m}, \quad(15)
\end{aligned}
$$

which hold for any test functions $v_{e}$ and $v_{m}$. The solution of (15) is equivalent to the original problem $P$.

\section{Projection method}

Using the projection method [10] let us reduce the variational equation (15) to a system of algebraic equations. Firstly, split an interval $\left[r_{0}, r\right]$ into $n$ subintervals with the length

$$
l=\frac{r_{0}-r}{n} .
$$

Let us define a set of $n$ subintervals

$$
\Phi_{i}=\left[r_{0}+(i-1) l, r_{0}+(i+1) l\right], i=1, \ldots, n-1
$$

and

$$
\Phi_{n}=\left[r_{0}+(n-1) l, h\right],
$$

and set of $n+1$ subintervals

$$
\begin{gathered}
\Psi_{1}=\left[r_{0}, r_{0}+l\right], \\
\Psi_{j}=\left[r_{0}+(i-2) l, r_{0}+i l\right], j=2, \ldots, n
\end{gathered}
$$

and

$$
\Psi_{n+1}=\left[r_{0}+(n-1) l, h\right] .
$$

These subintervals we call base finite elements.

In accordance with the scheme of the projection method, it is necessary to introduce basis functions $\phi_{i}$ and $\psi_{j}$ in order to approximate the solution. The basis functions are defined on each subinterval $\Phi_{i}$ and $\Psi_{j}\left(\phi_{i}\right.$ and $\psi_{j}$ vanishes outside the intervals $\Phi_{i}$ and $\Psi_{j}$, respectively). 
The basis functions $\phi_{i}$ define on $\Phi_{i}$, are

$\phi_{i}=\left\{\begin{array}{rl}\frac{\rho-r_{0}-(i-1) l}{l}, & \rho<r_{0}+i l, \\ -\frac{\rho-r_{0}-(i+1) l}{l}, & \rho>r_{0}+i l,\end{array}, i=\overline{1, n-1}\right.$

and

$$
\phi_{n}=\frac{\rho-h+l}{l} ;
$$

The basis functions $\psi_{i}$ defined on $\Phi_{i}$ are

$$
\begin{gathered}
\psi_{1}=-\frac{\rho^{2}-2 r_{0} \rho+r_{0}^{2}-l^{2}}{l^{2}}, \\
\psi_{2}= \begin{cases}\frac{\rho^{2}-2 r_{0} \rho+r_{0}^{2}}{l^{2}}, & \rho<r_{0}+l, \\
-\frac{\rho-r_{0}-2 l}{l}, & \rho>r_{0}+l,\end{cases} \\
\psi_{j}=\left\{\begin{array}{ll}
\frac{\rho-r_{0}-(i-2) l}{l}, & \rho<r_{0}+(i-1) l, \\
-\frac{\rho-r_{0}-i l}{l}, & \rho>r_{0}+(i-1) l,
\end{array}, \overline{3, n}\right.
\end{gathered}
$$

and

$$
\psi_{n+1}=\frac{\rho-h+l}{l} .
$$

Such defined basis functions takes into account the physical nature of the problem under consideration.

We assume an approximate solution with real coefficients $\alpha_{i}$ and $\beta_{j}$ such that

$$
u_{e}=\sum_{i=1}^{n} \alpha_{i} \phi_{i}, \quad u_{m}=\sum_{j=1}^{n+1} \beta_{j} \psi_{j} .
$$

Substituting functions $u_{e}$ and $u_{m}$ with representations (16) into the variational equation (15), we obtain a system of linear equations with respect to $\alpha_{i}$ and $\beta_{j}$ (for fixed value of $\gamma$ )

$$
A(\gamma) x=0,
$$

where matrices $A(\gamma)$ and $x$ have the form

$$
\begin{gathered}
A=\left(\begin{array}{cccccc}
A_{e e}^{1,1} & \cdots & A_{e e}^{1, n} & A_{e m}^{1,1} & \cdots & A_{e m}^{1, n+1} \\
\vdots & \ddots & \vdots & \vdots & \ddots & \vdots \\
A_{e e}^{n, 1} & \cdots & A_{e e}^{n, n} & A_{e m}^{n, 1} & \cdots & A_{e m}^{n, n+1} \\
A_{m e}^{1,1} & \cdots & A_{m e}^{1, n} & A_{m m}^{1,1} & \cdots & A_{m m}^{1, n+1} \\
\vdots & \ddots & \vdots & \vdots & \ddots & \vdots \\
A_{m e}^{n+1,1} & \cdots & A_{m e}^{n+1, n} & A_{m m}^{n+1,1} & \cdots & A_{m m}^{n+1, n+1}
\end{array}\right), \\
\quad x=\left(\begin{array}{c}
\alpha_{1} \\
\vdots \\
\alpha_{n} \\
\beta_{1} \\
\vdots \\
\beta_{n+1}
\end{array}\right),
\end{gathered}
$$

where

$$
\begin{aligned}
& A_{e e}^{i, j}=\gamma^{2} \int_{\Phi_{i}} h_{e} \phi_{i} \phi_{j} d \rho+ \\
& +\int_{\Phi_{i}} \phi_{i}^{\prime} \phi_{j}^{\prime} d \rho+\int_{\Phi_{i}} p_{e} \phi_{i}^{\prime} \phi_{j} d \rho-\int_{\Phi_{i}} q_{e} \phi_{i} \phi_{j} d \rho+ \\
& \quad+k_{1} \frac{\mu_{z}(r)}{\mu_{0}} \frac{K_{0}\left(k_{1} r\right)}{K_{1}\left(k_{1} r\right)} \phi_{i}(r) \phi_{j}(r), i, j=\overline{1, n} ; \\
& A_{e m}^{i, j}=\gamma \int_{\Phi_{i}} f_{m} \phi_{i} \psi_{j} d \rho, i=\overline{1, n}, j=\overline{1, n+1}, \\
& A_{m e}^{i, j}=\gamma \int_{\Psi_{i}} f_{e} \psi_{i} \phi_{j} d \rho, i=\overline{1, n+1, j=\overline{1, n},} \\
& +\int_{\Psi_{i}}^{i, j}=\gamma_{i}^{2} \int_{\Psi_{i}} \psi_{j}^{\prime} d \rho+\int_{\Psi_{i}} \psi_{i} \psi_{j} d \rho+ \\
& \quad+k_{1} \frac{\varepsilon}{\varepsilon_{0}} \frac{K_{0}\left(k_{1} r\right)}{K_{1}\left(k_{1} r\right)} \psi_{i}(r) \psi_{j} d \rho-\int_{\Psi_{i}}(r), i, j=\overline{1, n+1} q_{m} \psi_{i} \psi_{j} d \rho+
\end{aligned}
$$

Thus $A(\gamma)$ is a $(2 n+1) \times(2 n+1)$ matrix. Let us denote by $\Delta(\gamma)$ the determinant of $A(\gamma)$

$$
\Delta(\gamma)=\operatorname{det} A(\gamma)
$$

Note 2. If there exists $\gamma=\widetilde{\gamma}$ such that $\Delta(\widetilde{\gamma})=0$, then $\widetilde{\gamma}$ is an approximate spectral parameter of Problem $P$. In other words, if an interval $[\underline{\gamma}, \gamma]$ is such that $\Delta(\underline{\gamma}) \times$ $\Delta(\gamma)<0$, then this means that there exists $\gamma=\widetilde{\gamma} \in[\underline{\gamma}, \gamma]$ which is an spectral parameter of Problem P. This value can be calculated with any prescribed accuracy.

\section{Numerical results}

The results of the numerical solution of the problem of propagating electromagnetic waves of an anisotropic magnetic waveguide structure are presented. Numerical results are obtained with the help of the shooting method. Radii of the waveguide (internal and external) $r_{0}=2 \mathrm{~cm}, r=$ $4 \mathrm{~cm}$, permittivity $\varepsilon=4$. The values of the tensor components $\hat{\mu}$, are shown in the figure captions.

Numerical analysis of the behavior of dispersion curves (graphs of the dependence of the propagation constant $\gamma$ on the circular frequency $\omega$ ) is performed for the different components of tensor $\hat{\mu}$. In the case of $\mu_{\varphi} \rightarrow 0$, the number of hybrid modes coincides with the sum of the "polarized" modes (TE and TM), the dispersion curves for $\mu_{\varphi}=0$ (Fig. 2) coincide with the known dispersion curves for problems on propagating TE-and TM-polarized waves of an metaldielectric waveguide [11].

Figure 3 and Figure 4 shows the dispersion curves for the case when components of tensor $\hat{\mu}$ are functions. 


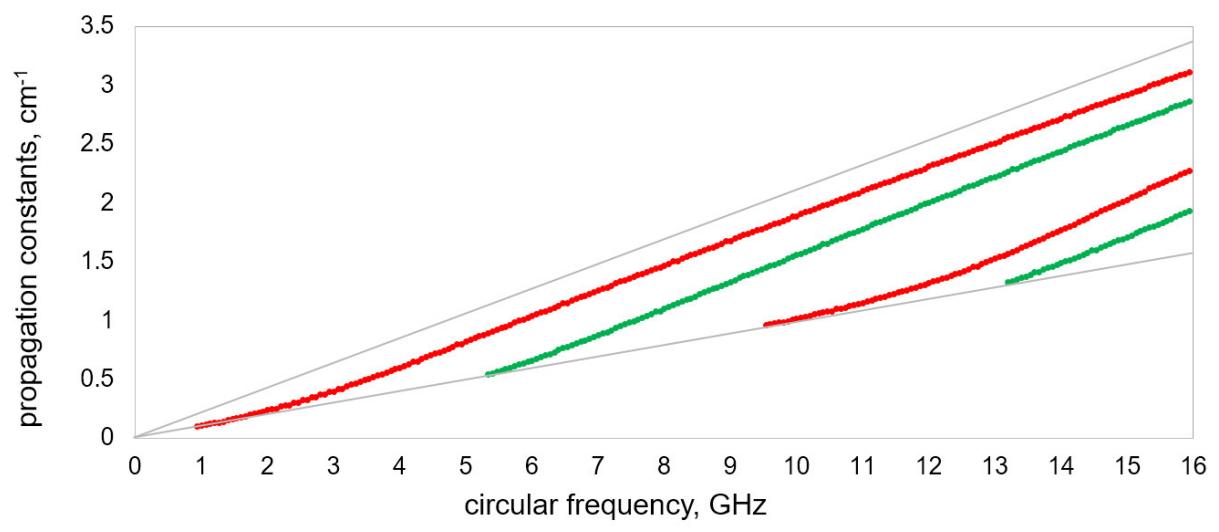

Figure 2: Dispersion curves for the values of the components of the tensor $\hat{\mu}: \mu_{\rho}=1, \mu_{z}=1 ; \mu_{\varphi}=0$. The red curves correspond to TM-polarized waves, the green curves to TE-polarized waves.

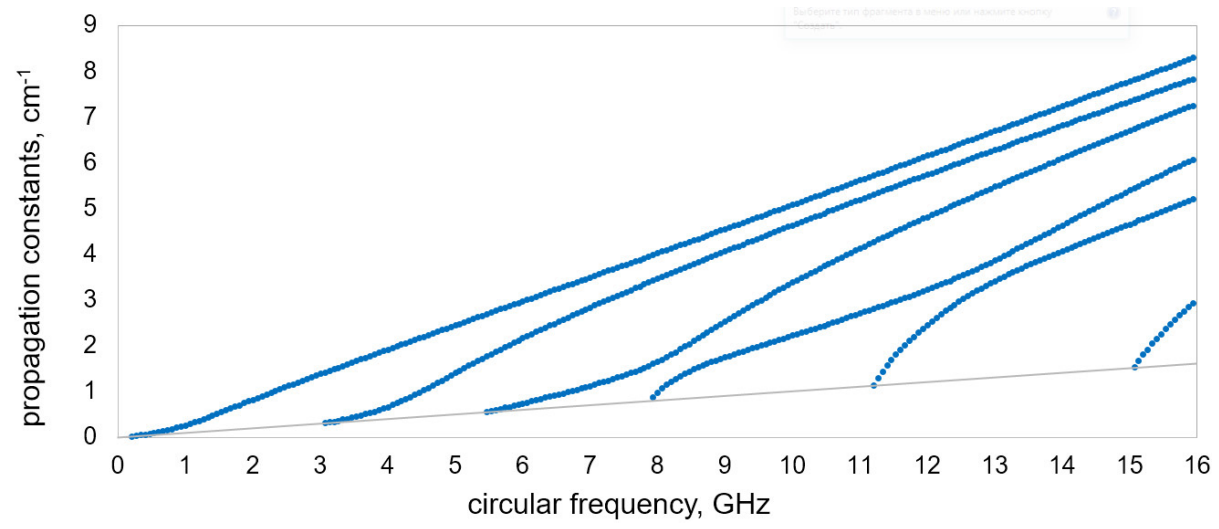

Figure 3: Dispersion curves for the values of the components of the tensor $\hat{\mu}$ : $\mu_{\rho}=1+\rho, \mu_{z}=1 ; \mu_{\varphi}=1$. The blue curves correspond to hybrid waves.

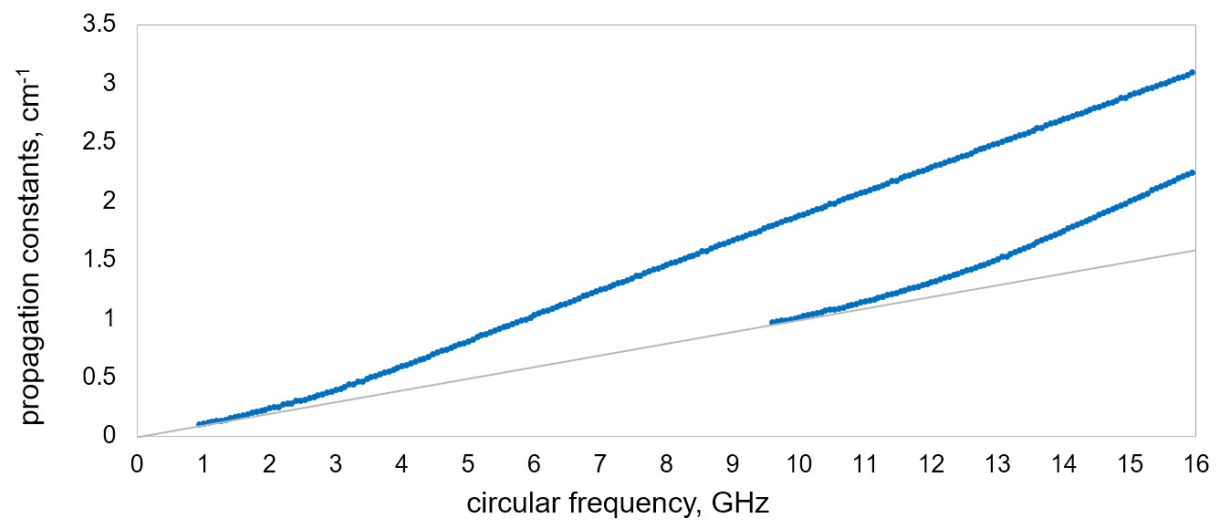

Figure 4: Dispersion curves for the values of the components of the tensor $\hat{\mu}: \mu_{\rho}=1, \mu_{z}=1+\rho ; \mu_{\varphi}=0.125$. The blue curves correspond to hybrid waves.

\section{Conclusion}

This work continues the investigation of the spectrum of metal dielectric waveguides with inhomogeneous filling. The paper[7] presents a numerical method for solving the problem of propagating waves of a dielectric waveguide. This method was used to numerically study the spectrum of a waveguide filled with an inhomogeneous anisotropic magnetic medium (ferrite). The method allows us to determine approximate eigenvalues with any prescribed accu- 
racy. The approach described in this paper can be applied to other problems, e.g., to multilayered opened waveguides.

\section{Acknowledgement}

This study is supported by the Ministry of Education and Science of the Russian Federation, Project No. 1.894.2017/4.6.

\section{References}

[1] L. Lewin, Theory of waveguides: Techniques for the solution of waveguide problems, New York, Halsted Press, 1975.

[2] L. A. Vainshtein, Electromagnetic Waves, Radio i Svyaz, Moscow, 1988. [in Russian].

[3] G. Lifante, F. Cusso and E. Cantelar, Numerical methods for optical waveguide devices, 2006 International Conference on Mathematical Methods in Electromagnetic Theory, Kharkiv, 2006, pp. 77-82.

[4] S. M. Saad, Review of Numerical Methods for the Analysis of Arbitrarily-Shaped Microwave and Optical Dielectric Waveguides, IEEE Transactions on Microwave Theory and Techniques, vol. 33, no. 10, pp. 894-899, Oct 1985.

[5] R. K. Varshney, I. C. Goyal and A. K. Ghatak, A simple and efficient numerical method to study propagation characteristics of nonlinear optical waveguides, Journal of Lightwave Technology, vol. 16, no. 4, pp. 697702, Apr 1998.

[6] J. C. Baumert and J. Hoffnagle, Numerical method for the calculation of mode fields and propagation constants in optical waveguides, Journal of Lightwave Technology, vol. 4, no. 11, pp. 1626-1630, Nov 1986.

[7] E. Smolkin, Numerical Method for Electromagnetic Wave Propagation Problem in a Cylindrical Inhomogeneous Metal Dielectric Waveguiding Structures, Mathematical Modelling and Analysis, Vol. 22, Issue 03, pp. $271-282,2017$.

[8] A.W. Snyder, J. Love, Optical waveguide theory, Springer, 1983.

[9] A. F. Nikiforov, V. B. Uvarov Special Functions of Mathematical Physics: A Unified Introduction with Applications, Nauka, 1978.

[10] D. Colton, R. Kress, Integral Equation Methods in Scattering Theory, Wiley, New York, 1984.

[11] E. Smolkin, Y., Shestopalov, Nonlinear Goubau line: analytical-numerical approaches and new propagation regimes, Journal of Electromagnetic Waves and Applications, 31 (8), pp. 781-797, 2017. 